\title{
Die Wandlung des Grundrechtsschutzes durch das Bundesverfassungsgericht - Recht auf Vergessen I und II als „Solange III"?*
}

\begin{abstract}
The relationship between the fundamental rights as laid down in the German Constitution and the fundamental rights contained in the Charter of Fundamental Rights of the European Union has been exceedingly conflict-prone ever since the early days of the European Union. Related thereto is the ongoing controversy on the judicial prerogative of the German Federal Constitutional Court (BVerfG) within that system. Thus, two big players in the European multi level system, i.e. the BVerfG and the European Court of Justice (ECJ), clash with their judicial powers and diverging interests.

With its two recent decisions, 1 BvR 16/13 and 1 BvR 276/17 of November 6, 2019, the First Senate of the BVerfG introduced a far-reaching change in its approach of protecting basic rights by clarifying the relationship between the EU fundamental rights and the fundamental rights of the German Constitution. At the same time, the $B V e r f G$ has made a strong effort to maintain its position within the multilevel cooperation of the constitutional courts of EU member states, particularly in relation to the ECJ, which by both sides is referred to as a "cooperative relationship".

This article explains the repercussions of the aforementioned judgments on the protection of fundamental rights in the European multi-level system.
\end{abstract}

\section{Résumé}

La relation entre les droits fondamentaux garantis dans la Constitution allemande et ceux énumérés dans la Charte des droits fondamentaux de l'Union européenne (UE) est extrêmement conflictuelle et ce depuis les débuts de l'UE. Liée à cela est la polémique actuelle sur la prérogative judiciaire de la Cour constitutionnelle fédérale allemande (BVerfG) au sein de ce système. Ainsi, deux grands acteurs du système européen à multiples niveaux, à savoir le BVerfG et la Cour de justice de l'Union européennes (CJUE), s'affrontent avec leurs pouvoirs judiciaires et leurs intérêts divergents.

Avec ses deux décisions récentes " 1 BvR 16/13 » et " 1 BvR 276/17 》 du 6 novembre 2019, le premier Sénat de la BVerfG a instauré un changement profond dans son approche de la protection des droits fondamentaux en clarifiant la relation entre les droits fondamentaux de l'UE et ceux de la Constitution allemande. Dans le même temps, le BVerfG a fait tout son possible pour maintenir sa position au sein de la

* Prof. Dr. Dr. Rainer Hofmann ist Inhaber des Lehrstuhls für Öffentliches Recht, Völkerrecht und Europarecht an der Goethe-Universität Frankfurt am Main. Alexander Heger ist dort wiss. Mitarbeiter und Tamara Gharibyan stud. Hilfskraft. 
coopération à plusieurs niveaux des cours constitutionnelles européennes, en particulier en ce qui concerne la CJUE. Cette relation est considerée par les deux parties comme une "relation de coopération".

Cet article explique les répercussions des arrêts susmentionnés sur la protection des droits fondamentaux des personnes dans le système européen à multiples niveaux.

\section{Zusammenfassung}

Das Verhältnis zwischen den Grundrechten des Grundgesetzes und den Grundrechten der Europäischen Union (EU) und der damit verbundene Konflikt um die (Kontroll-) Kompetenzen des Bundesverfassungsgerichts (BVerfG), ist schon seit den Anfangszeiten der EU höchst spannungsgeladen. Neben dem Verhältnis der Rechtsordnungen begegnen sich zwei Höchstgerichte, BVerfG und Europäischer Gerichtshof (EuGH), mit ihren gerichtlichen Kompetenzen und unterschiedlichen Interessen.

Mit den beiden jüngsten Entscheidungen 1 BvR 16/13 (Recht auf Vergessen I) sowie 1 BvR 276/17 (Recht auf Vergessen II) vom 06. November 2019 hat der Erste Senat des BVerfG eine weitreichende Veränderung des System des Grundrechtsschutzes vorgenommen, indem er das Verhältnis zwischen den Unionsgrundrechten und den Grundrechten des Grundgesetzes weitestgehend konkretisierte.

Zugleich hat das BVerfG die Anstrengung unternommen, seinen Platz im Europäischen Verfassungsgerichtsverbund (insbesondere im Verhältnis zum EuGH) zu behaupten, welches von beiden Seiten als sog. „Kooperationsverhältnis“ verstanden wird. Dieser Aufsatz legt dar, welche Auswirkungen die Urteile des BVerfG auf den Grundrechtsschutz im Mehrebenensystem haben.

\section{Grundrechtsverbürgungen im Mehrebenensystem}

An sich existieren - neben den landesverfassungsrechtlichen Grundrechtsverbürgungen - die Grundrechte der EMRK, der Grundrechte Charta (GRCh) sowie die des Grundgesetzes (GG). Zwar kann sich der Bürger vor dem BVerfG nicht unmittelbar auf die Verbürgungen der EMRK berufen, indes sind die Grundrechte des Grundgesetzes - nach ständiger Rspr. des BVerfG - im Lichte der EMRK auszulegen. ${ }^{1}$ Hierarchisch oberhalb der EMRK - welche einfachgesetzlichen Rang genießt - stehen sodann die Grundrechte des Grundgesetzes sowie die der GRCh. Die Grundrechte des Grundgesetzes binden die deutsche Staatsgewalt grundsätzlich umfassend, Art. 1 Abs. 3 GG. ${ }^{2}$ Im Umkehrschluss bedeutet dies jedoch, dass andere Hoheitsgewalt (wie die der Europäischen Union) aufgrund deren Autonomie von dieser Bindung nicht betroffen ist. ${ }^{3}$

1 BVerfGE 111, 307 (317) - Görgülü; BVerfGE 131, 286 (295) - Sicherungsverwahrung II.

2 Vgl. auch Bäcker EuR 2015, 389 (390).

3 BeckOK Grundgesetz/Hillgruber, 41. Ed. (2019), Art. 1 GG, Rn. 80, vgl. auch BVerfGE 58, $1(27)$. 
Gleichwohl besteht eine fortschreitende Europäisierung des Rechts, welche die Frage aufwirft, welchen Stellenwert dabei die deutsche Staatsgewalt und die Grundrechte des Grundgesetzes noch haben. Zumindest in eine Richtung hat der Verfassungsgeber Ende 1992 mit der Einführung des Art. 23 GG vorgegeben, dass sich die deutsche Staatsgewalt nicht dadurch ihren grundrechtlichen Bindungen entzieht, dass sie Kompetenzen an eine supranationale Organisation abgibt und damit den Regelungsbereich der deutschen Staatsgewalt reduziert. Auf dieser supranationalen Ebene muss ein im Wesentlichen dem Grundgesetz vergleichbarer Grundrechtsschutz gewährleistet sein (vgl. Art. 23 Abs. 1 S. 1 GG). ${ }^{4} \mathrm{Zu}$ klären bleibt jedoch, in welchem Verhältnis die Grundrechtsverbürgungen zueinander stehen, insbesondere dann, wenn sowohl die deutsche als auch supranationale Hoheitsgewalt betroffen sind.

\section{Entwicklung der Grundrechte in der Europäischen Union}

Im Grundgesetz stehen die Grundrechtsverbürgungen schon seit 1949 an prominenter Stelle, nämlich am Beginn des Grundgesetzes. Die Gemeinschaft kannte jedoch zunächst keine geschriebenen Grundrechte. Eine Ausnahme war nur in den Diskriminierungsverboten zu erblicken (Art. 12 und 13 EG a.F). Das bedeutete jedoch nicht, dass es innerhalb der Gemeinschaft keine Grundrechte gab. Diese Aufgabe zur Schaffung von Grundrechtsverbürgungen übernahm der EuGH. In seinem Urteil in der Rechtssache Stauder erkannte er den Grundrechtsschutz an, ${ }^{5}$ deren Gehalt zukünftig aus einer wertenden Rechtsvergleichung der mitgliedstaatlichen Verbürgungen unter Berücksichtigung der EMRK bestimmt wurde. ${ }^{6} 1999$ wurde ein Grundrechtekonvent errichtet, der den Beschluss des Europäischen Rates umsetzen sollte, eine Europäische Grundrechte-Charta zu schaffen. ${ }^{7}$ Diese wurden zunächst jedoch ohne Bindungswirkung proklamiert. ${ }^{8}$ Mit den Vertragsrevisionen von Lissabon ist die GRCh jedoch zu Primärrecht erhoben worden (Art. 6 I EUV).

\section{Entwicklung der Judikatur des BVerfG zu dem Verhältnis natio- naler Grundrechte zur GRCh}

In der Frühphase der europäischen Integration behielt sich das BVerfG noch die umfassende Kontrolle von Unionsrechtsakten anhand der nationalen Grundrechte vor. In seiner Solange I-Entscheidung von 1974 betonte der Zweite Senat des BVerfG, dass, solange die Gemeinschaft über keinen dem Grundgesetz adäquaten Grundrechtskatalog verfügt, eine Kontrolle von sekundärem Gemeinschaftsrecht am Maßstab der nationalen Grundrechte durch das BVerfG erfolgen und ihnen ihre Wirkung im nationa-

4 BeckOK Grundgesetz/Hillgruber, 41. Ed. (2019), Art. 1 GG, Rn. 80.

5 EuGH, v. 12.11.1969 - Rs. 29/69, ECLI:EU:C:1969:57 - Stauder.

6 EuGH v. 14.5.1974 - Rs. 4/73, ECLI:EU:C:1974:51 - Nold.

7 http://www.europarl.europa.eu/summits/kol2_de.htm.

8 auch BVerfGE 123, 267 (277). 
len Rechtsraum abgesprochen werden kann, sofern sie mit den Grundrechten des Grundgesetzes kollidieren. ${ }^{9}$

Dieser Beschluss lässt sich nur vor dem Hintergrund der bis dato mühsamen Entwicklung des Grundrechtsschutzes auf Unionsebene verstehen. Zwar hatte der EuGH im selben Jahr mit der Entscheidung Nold ${ }^{10}$ tatsächlich mit dem Aufbau einer eigenen Grundrechtsordnung begonnen, doch wollte sich das BVerfG, angesichts des zuvor ergangenen, inhaltlich vergleichbaren Urteils des italienischen Verfassungsgerichtshofs nicht nehmen lassen, seinen eigenen Standpunkt deutlich kund zu tun. Schließlich gestatte die Integrationsermächtigung keine beliebige Relativierung der Grundrechte als Elemente der Verfassungsstruktur. ${ }^{11}$

Mit fortschreitender Integration erhöhte sich in der Folge auch der Grundrechtsschutz. Insbesondere aufgrund der zunehmenden Rechtsprechung des EuGH zu den Grundrechten als allgemeine Rechtsgrundsätze, der Bezugnahme zu dem Grundrechtskatalog der EMRK, sowie der Herausarbeitung allgemeiner Grundrechtslehren, ${ }^{12}$ änderte sich die Rechtsprechung des BVerfG im Zuge der Solange-II-Entscheidung von 1986. Demnach übt das BVerfG seine Gerichtsbarkeit nicht mehr aus, solange die Gemeinschaft, insbesondere durch die Rechtsprechung des EuGH, einen wirksamen Schutz der Grundrechte generell gewährleistet, der dem vom Grundgesetz als unabdingbar gebotenen Schutz im Wesentlichen gleichzusetzen ist. ${ }^{13} \mathrm{Zu}$ beachten bleibt jedoch, dass das BVerfG seine Prüfungskompetenz damit nicht aufgegeben, sondern in Anbetracht des von ihm als angemessen qualifizierten Grundrechtsschutzes durch den EuGH nur bis auf Weiteres zurückgenommen oder bis auf Weiteres suspendiert hat: ${ }^{14}$ Es hat nämlich erklärt, dass seine grundsätzlich auch weiterhin bestehende Befugnis (und Pflicht) sicherzustellen, dass im Geltungsbereich des Grundgesetzes keine (gemeinschaftsrechtlichen) Normen zur Anwendung kommen, denen gegenüber kein Grundrechtsschutz besteht, der mit dem vom Grundgesetz als unabdingbar verlangten Grundrechtsschutz im Wesentlichen vergleichbar sei, solange nicht mehr auszuüben, solange ein solcher qualifizierter Grundrechtsschutz vom EuGH gewährleistet werde. ${ }^{15}$

Den vorübergehenden Endpunkt dieser Rechtsprechung stellte die sog. Bananenmarkt-Entscheidung aus dem Jahre 2000 dar. Demnach muss bei Geltendmachung einer Grundrechtsverletzung durch Sekundärrecht im Einzelnen dargelegt werden, dass der jeweils als unabdingbar gebotene Grundrechtsschutz auf Unionsebene generell nicht gewährleistet ist. Andersfalls seien entsprechende Verfassungsbeschwerden und Richtervorlagen von vornherein unzulässig. ${ }^{16}$ Ein einmaliges Absinken reicht al-

9 BVerfGE 37, 271 (285) - Solange I.

10 EuGH v. 14.5.1974 - Rs. 4/73, ECLI:EU:C:1974:51 - Nold.

11 BVerfGE 37, 271 (280).

12 Siehe insbes. EuGH, Rs. 44/79, ECLI:EU:C:1979:290 - Hauer.

13 BVerfGE 73, 339 (387) - Solange II.

14 Dies bestätigte das BVerfG auch im Vorratsdaten-Urteil vom 2.3.2010, BVerfGE 125, 260.

15 Vgl. hierzu Hofmann, Zurück zu Solange II! Der Bananenmarktordnungs-Beschluß des Bundesverfassungsgerichts, in: Cremer u.a. (Hrsg.), Tradition und Weltoffenheit des Rechts, Festschrift für Helmut Steinberger, S. 1207 (1210).

16 BVerfG 102, 147 (166) - Bananenmarktordnung; vgl. hierzu Hofmann (Fn. 15), 1207 (1217). 
so gerade nicht aus, um eine Überprüfung anhand nationaler Grundrechte zu bewirken. ${ }^{17}$

Dieser Grundsatz galt jedoch nicht nur für das Sekundärrecht selbst, sondern - nach bisheriger Rspr. des BVerfG - auch für nationale Umsetzungsmaßnahmen (z.B. Gesetze zur Richtlinienumsetzung), soweit das Unionsrecht keine Umsetzungsspielräume zulässt. ${ }^{18}$ Hintergrund ist, dass eine solche nationale Regelung, bei materieller Betrachtung als EU-Recht im nur formalen Gewande des nationalen Rechts erscheint. ${ }^{19}$ Eine Prüfung anhand der Grundrechte des Grundgesetzes käme in einem solchen Fall allenfalls entsprechend den Vorgaben des Solange-Vorbehalts in Betracht.

Hingegen sollen die Grundrechte des Grundgesetzes im Falle der Ausfüllung eines Gestaltungsspielraums - bezüglich dieser Ausfüllung - vollumfänglich zur Anwendung gelangen, ${ }^{20}$ sodass im Ergebnis eine Art „Trennungstheorie“ begründet wurde, wonach im Bereich vollharmonisierter Vorgaben die EU-Grundrechte zur Anwendung gelangen, während im Spielraumbereich die nationalen Grundrechte gelten.

Eine Besonderheit gilt seit dem Lissabon-Urteil ${ }^{21}$ des BVerfG bei der Geltendmachung einer Menschenwürdeverletzung bzw. der Verfassungsidentität des Grundgesetzes, die ihren Ausgangspunkt in Art. 79 Abs. 3 GG nimmt. Hierbei bedarf es nicht eines generellen Absinkens des Grundrechtsschutzes auf Unionsebene, um eine Prüfung am Maßstab der nationalen Grundrechte auszulösen; vielmehr wird eine einzelfallbezogene Prüfung angestellt, wenn dies zur Wahrung der durch Art. 79 Abs. 3 i.V.m. Art. 1 und Art. 20 GG verbürgten Verfassungsidentität unabdingbar geboten ist. Dies ist deshalb vom Solange-Vorbehalt zu trennen. Deshalb spielt es also keine Rolle, inwiefern der Sachverhalt unionsrechtlich determiniert ist, sofern die Menschenwürde unmittelbar oder mittelbar als Kerngehalt eines anderen Grundrechts berührt ist. $\mathrm{Zu}$ den Schutzgütern der in Art. 79 Abs. 3 GG niedergelegten Verfassungsidentität, die auch vor Eingriffen durch die supranational ausgeübte öffentliche Gewalt geschützt sind, gehören die Grundsätze des Art. 1 GG, also die Verpflichtung aller staatlichen Gewalt, die Würde des Menschen zu achten und zu schützen (Art. 1 Abs. 1 Satz 2 GG). ${ }^{22}$ Die Rechtsprechung zur Identitätskontrolle wurde durch die Entscheidungen in OMT-Verfahren sowie PSPP-Verfahren vor dem BVerfG konkretisiert. Aus Sicht des BVerfG beinhaltet das Wahlrecht des Einzelnen aus Art. 38 Abs. 1 Satz 1 GG neben der formalen Legitimation der (Bundes-) Staatsgewalt auch dessen grundlegenden demokratischen Gehalt, der insbesondere das Recht der Bürger gewährleistet, an der demokratischen Willensbildung durch die Mitwirkung an den Wahlen des Bundestages teilzunehmen, und zugleich eine Entleerung dieses Rechts verbietet. ${ }^{23}$ Dieser „Anspruch auf Demokratie“24 ist allerdings strikt auf den in der Würde des Menschen wurzelnden Kern des Demokratieprinzips begrenzt (Art. 1 i.V.m. Art. 79 Abs. 3 GG).

17 Ludwigs, EuGRZ, 2014, 273 (274).

18 BVerfGE 118, 79 (95) - TEHG.

19 Ludwigs/Sikora, EWS 2016, 121 (124).

20 BVerfGE 125, 260 (306f.) - Vorratsdatenspeicherung.

21 BVerfGE 123, 267 - Lissabon.

22 BVerfGE 140, 317 (341) - Europäischer Haftbefehl II.

23 vgl. BVerfGE 89, 155 (171 f.); BVerfGE 146, 216 (249) - PSPP.

24 BVerfGE 142, 123 (219) - OMT II; BVerfG v. 30.7.2019 - 2 BvR 1685/14 (Bankenunion), Rn. 118. 
Art. 38 Abs. 1 Satz 1 GG gewährt keinen Anspruch auf eine über dessen Sicherung hinausgehende Rechtmäßigkeitskontrolle demokratischer Mehrheitsentscheidungen. Er dient nicht der inhaltlichen Kontrolle demokratischer Prozesse, sondern ist auf deren Ermöglichung gerichtet. ${ }^{25}$ Da der deutsche Gesetzgeber bei der Übertragung von Kompetenzen an die Union diese Verfassungsgarantien nicht hätte einschränken können, dürfen auch die Hoheitsakte der Union diese Garantien nicht verletzen. ${ }^{26}$ Das Grundgesetz ermächtigt die deutschen Staatsorgane auch nicht, Hoheitsrechte derart zu übertragen, dass aus ihrer Ausübung heraus eigenständig weitere Zuständigkeiten für die Europäische Union begründet werden können ${ }^{27}$ oder den unantastbaren Kern des Grundgesetzes verletzen. ${ }^{28}$ Indem das BVerfG die demokratische Legitimation der in Deutschland ausgeübten öffentlichen Gewalt durch das Staatsvolk als wesentlichen Inhalt des Grundsatzes der Volkssouveränität bezeichnet, der durch Art. 79 Abs. 3 GG und Art. 23 Abs. 1 Satz 3 einen Teil der integrationsfesten Verfassungsidentität des Grundgesetzes darstellt, verknüpft es die ultra-vires-Kontrolle mit der Identitätskontrolle. ${ }^{29}$ Das BVerfG prüft also im Rahmen der Identitätskontrolle, ob die Übertragung von Hoheitsrechten unter Preisgabe des über Art. 79 Abs. 3 GG in Verbindung mit Art. 23 Abs. 1 Satz 3 GG geschützten Inhalts des Grundsatzes der Volkssouveränität (Art. 20 Abs. 1 und Abs. 2 Satz 1 GG) erfolgte. ${ }^{30}$ Art. 38 Abs. 1 Satz 1 GG verbürgt deutschen Staatsbürgern ein Recht auf demokratische Selbstbestimmung, sofern sich das Handeln der EU bzw. ihrer Organe außerhalb der über Art. 23 GG übertragenen Kompetenzen bewegt. ${ }^{31}$

In dem Beschluss 2 BvR 2735/14 vom 15.12.2015, in dem es um die Auslieferung eines in Abwesenheit Verurteilten ging, entschied das BVerfG, dass der in der Menschenwürdegarantie des Art. 1 Abs. 1 GG verankerte Grundsatz, dass jede Strafe Schuld voraussetzt, zur Verfassungsidentität gehört. ${ }^{32}$ Die vom Grundgesetz als integrationsfest erklärten Grundsätze bilden damit eine Grenze für den Anwendungsvorrang des Unionsrechts. ${ }^{33}$ Im Falle des Beschlusses 2 BvR 2735/14 erfolgte jedoch keine Anwendung der Identitätskontrolle. Die Notwendigkeit unter Rückgriff auf Art. 79 Abs. 3 GG iVm Art. 1 Abs. 1 GG den dem Rahmenbeschluss zukommenden Anwendungsvorrang zu begrenzen, sah das BVerfG vorliegend ,jedoch nicht, weil sowohl der Rahmenbeschluss selbst (a) als auch das diesen umsetzende Gesetz über die internationale Rechtshilfe in Strafsachen (b) eine Auslegung gebiete, die den von Art. 1 Abs. 1 GG geforderten Mindestgarantien von Beschuldigtenrechten bei einer Auslieferung Rechnung“ trüge. ${ }^{34}$

25 BVerfGE 146, $216(249)$ - PSPP.

26 Vgl. BVerfGE 113, 273 (296); 123, 267 (348); 134, 366 (384)).

27 BVerfGE 142, 123 (174 f.) - OMT II.

28 BVerfGE 134, 366), 382 f) - OMT I; BVerfGE 140, 317 (336f.) - Europäischer Haftbefehl II.

29 So schon: Ludwigs/Sikora, EWS 2016, 215 (219).

30 BVerfG v. 30.7.2019 - 2 BvR 1685/14 (Bankenunion), Rn. 119 f.

31 BVerfGE 146, 216 (249) - PSPP.

32 BVerfGE 140, 317 (341 f.) - Europäischer Haftbefehl II.

33 Kritik zur Definition der Grundsätze u.a. Ludwigs/Sikora, EWS 2016, 121 (129); Schwerdtfeger, EuR 2015, 290 (293 f.).

34 BVerfGE 140, 317 (355) - Europäischer Haftbefehl II. 
Sofern also verfassungs- und unionsrechtlichen Anforderungen identisch sind, spielt der Anwendungsvorrang des Unionsrechts hier keine Rolle, weil es schon an einer Kollision zwischen Unions- und Verfassungsrecht fehlt. Unionsrechtliche Determinierung deutschen Staatshandelns hindert, auch wenn sie abschließend ist, eine Aufhebung gerichtlicher Entscheidungen unter verfassungsrechtlichen Gesichtspunkten nicht, wenn diese die Anordnungen des Unionsrechts nicht in Frage stellt, sondern geradezu verwirklicht. ${ }^{35}$

Die Identitätskontrolle bildet jedoch einen Konfliktpunkt zur Solange-Rechtsprechung, sofern spezifisch grundrechtsrelevante Ausprägungen betroffen sind. Mit der im Endeffekt nicht erfolgten, ${ }^{36}$ aber angedrohten - Anwendung der Identitätskontrolle, hat das BVerfG jedoch schon mit der Entscheidung zum Europäischen Haftbefehl deutlich gemacht, von der generellen Rücknahme seiner grundrechtlichen Kontrollzuständigkeit abrücken zu können oder sogar zu wollen.

\section{Entscheidungen des BVerfG}

Entsprechend des bisherigen Verständnisses ist es also für die Frage der Anwendbarkeit der Grundrechte des Grundgesetzes maßgeblich, ob das relevante Unionsrecht vollständig harmonisierenden Charakter hat (i.d.R. bei Verordnungen zu bejahen) oder den Mitgliedstaaten Gestaltungsfreiheiten belässt (i.d.R. bei der Umsetzung von Richtlinien zutreffend). ${ }^{37}$

Vor diesem Hintergrund und der Tatsache, dass den Beschlüssen des BVerfG jeweils Rechtsstreitigkeiten zugrunde lagen, die einerseits eine unionsrechtlich abschließend determinierte Regelung betrafen, andererseits jedoch eine Materie gegenständlich war, welche zwar im Anwendungsbereich des Unionsrechts lag, jedoch von den Mitgliedstaaten autonom ausgestaltet werden durfte, konnte das BVerfG in den vorgenannten Grundsatzentscheidungen seine durch die Rechtsprechung gefestigten Reglements weiter konkretisieren.

\section{Kein Gestaltungsspielraum zugunsten der Mitgliedstaaten}

Existiert bei dem umzusetzenden und zu vollziehenden Unionsrecht kein Gestaltungsspielraum zugunsten der Mitgliedstaaten, kann dieser Akt nicht anhand von Grundrechten des Grundgesetzes überprüft werden. Dies ist letztlich Konsequenz des Anwendungsvorranges von Unionsrecht und entspricht dem bisherigen Verständnis, welches jüngst von dem BVerfG bestätigt wurde. ${ }^{38}$ Kämen die Grundrechte des Grundgesetzes auch gegenüber dem Unionsrecht zur Anwendung, wäre eine einheitliche An-

35 BVerfGE 140, 317 (352ff.) - Europäischer Haftbefehl II; so auch Ruffert, JuS 2016, 373 (375); Ludwigs/Sikora, EWS 2016, 121 (128). Kritisch, ob in diesem Fall überhaupt eine unionsrechtliche Determinierung vorliegt, Schönberger, JZ 2016, 422.

36 Dabei ist es egal, ob die Anwendung der Identitätskontrolle nicht notwendig oder sogar gar nicht möglich war.

37 BVerfG, Beschluss des Ersten Senats vom 06. November 2019 - 1 BvR 276/17, Rn. 77.

38 BVerfG, a.a.O., Rn. 41. 
wendung des Unionsrechts nicht mehr gewährleistet. Hierbei sei jedoch auf die vom BVerfG entwickelten „Grenzen“ der sog. Identitäts- und ultra-vires-Kontrolle verwiesen, welche ihrerseits hingegen nicht kritiklos ${ }^{39}$ hingenommen werden. Für den Fall der Nichtanwendbarkeit der Grundrechte des Grundgesetzes gelten folglich die Grundrechte der GRCh. Bisher erfolgte die Wahrung der Unionsgrundrechte in Kooperation durch die Fachgerichte mit dem EuGH. Ein Bürger konnte bis dato nur vor das BVerfG ziehen, wenn er eine Verletzung des Grundrechts auf den gesetzlichen Richter (Art. 101 I 2 GG) in Form der Verletzung der Vorlagepflicht seitens letztinstanzlicher Fachgerichte (vgl. Art. 267 III AEUV) geltend machte. Darüber hinaus musste er entweder ein generelles Absinken des Grundrechtsschutzes auf Unionsebene (Solange-Rechtsprechung), eine Verletzung der Verfassungsidentität (Identitätskontrolle) oder ein kompetenzüberschreitendes Handeln der Unionsorgane (ultra-vires-Kontrolle) darlegen, um eine verfassungsgerichtliche Kontrolle herbeizuführen.

Das BVerfG entschied jedoch nun, dass es selbst im Rahmen einer Verfassungsbeschwerde auch die Grundrechte der GRCh als unmittelbaren Prüfungsmaßstab heranziehen kann. ${ }^{40}$ Damit schließt sich das BVerfG der Vorgehensweise des österreichischen, italienischen als auch belgischen Verfassungsgerichtes an. ${ }^{41}$

Soweit also die Grundrechte des Grundgesetzes durch den Anwendungsvorrang des Unionsrechts verdrängt werden, kontrolliert das BVerfG die Anwendung des Unionsrechts durch deutsche Stellen am Maßstab der Unionsgrundrechte. ${ }^{42}$

Das BVerfG begründet dies mit der Integrationsverantwortung, welche aus Art. 23 Abs. 1 GG erwachse, sowie seinem verfassungsrechtlichen Auftrag zum Grundrechtsschutz. Zwar war die u.a. durch Art. 93 Abs. 1 Nr. 4 a GG dem BVerfG zugewiesene Kompetenz zur Gewährleistung eines Grundrechtsschutzes ursprünglich nur auf deutsche Grundrechte ausgerichtet. Die Grundrechte aus der GRCh seien jedoch funktionsäquivalent zu denen des Grundgesetzes, da auch die GRCh dem Schutz der Freiheit und Gleichheit der Bürger diene. ${ }^{43}$ Ohne Prüfungskompetenz des BVerfG sei der Rechtsschutzapparat unvollständig und berge Schutzlücken, da dieses auch gegenüber den Fachgerichten seine grundrechtsspezifische Kontrollfunktion wahrnehmen müsse. ${ }^{44}$ Dies gilt insbesondere deshalb, weil kein effektiver Individualrechtsbehelf hinsichtlich der GRCh existiert. ${ }^{45}$

Prozessual bedeutet dies, dass mit der Verfassungsbeschwerde auch eine Verletzung von Rechten der GRCh geltend gemacht werden können.

Zutreffend erkennt das BVerfG jedoch auch, dass die letztverbindliche Auslegung der Grundrechte der GRCh allein dem EuGH zusteht. In diesem Zusammenhang betont das BVerfG die Vorlagepflichtigkeit des BVerfG bei Zweifeln hinsichtlich der Reichweite der dort garantierten Grundrechte (Art. 276 Abs. 3 AEUV). Unberührt bleiben ebenfalls Vorlagerechte der Fachgerichte und deren Verpflichtung, die Grund-

39 Ludwigs/Sikora, EWS 2016, 121.

40 BVerfG, a.a.O., Rn. $50 \mathrm{ff}$.

41 Vgl. BVerfG, a.a.O., Rn. 50.

42 BVerfG, a.a.O., Rn. 51.

43 BVerfG, a.a.O., Rn. 59.

44 BVerfG, a.a.O., Rn. 62.

45 BVerfG, a.a.O., Rn. 61. 
rechte der Europäischen Union zu beachten. Dieses Bewusstsein ist für das Kooperationsverhältnis zum EuGH von elementarer Bedeutung.

\section{Gestaltungsspielraum zugunsten der Mitgliedstaaten}

In seiner zweiten Entscheidung bestätigt das BVerfG im Grundsatz sein bisheriges Verständnis, konkretisiert es jedoch weiter. Handelt es sich um Unionsrecht, welches Spielräume zugunsten der Mitgliedstaaten vorsieht und gerade nicht vollständig harmonisierend ist, bleibt eine Überprüfung der Ausübung und Anwendung des Gestaltungsspielraums anhand der Grundrechte des Grundgesetzes möglich, da deutsche Staatsgewalt an die Grundrechte des Grundgesetzes gebunden ist. Diese Vorgehensweise hat auch der EuGH bestätigt, wonach im Falle dieser Umsetzungsspielräume diese durch das nationale Verfassungsrecht - insbesondere die nationalen Grundrechte - eingeschränkt werden können, ohne dass dies unionsrechtlich bedenklich wäre. ${ }^{46}$ In diesem Fall hat das BVerfG die Beachtung der Grundrechte des Grundgesetzes zu gewährleisten. ${ }^{47}$ Untermauert wird dies dadurch, dass die EU Grundrechtsvielfalt zulässt. ${ }^{48}$ Folglich bleibt es bei dem Grundsatz, dass das BVerfG innerstaatliches Recht und dessen Anwendung auch am Maßstab des Grundgesetzes prüft, selbst wenn es im Anwendungsbereich von Unionsrecht liegt, nicht aber vollständig determiniert ist. ${ }^{49}$

Das BVerfG entschied jedoch auch, dass trotz Gestaltungsspielraum nicht ausgeschlossen sei, dass neben der Anwendung der Grundrechte des Grundgesetzes im Einzelfall auch die GRCh Geltung beanspruchen kann. ${ }^{50}$ In Betracht kommt das nur im Rahmen der unionsrechtlichen Verträge und damit dann, wenn nach Art. 51 Abs. 1 Satz 1 GRCh die „Durchführung von Unionsrecht“ in Frage steht. ${ }^{51}$ Dies ist vor allem für die Fachgerichte relevant. Das BVerfG stellt mit dieser Entscheidung zugleich auch eine widerlegliche Vermutung dafür auf, dass durch eine Prüfung am Maßstab der Grundrechte des Grundgesetzes das Schutzniveau der GRCh, wie sie vom Europäischen Gerichtshof ausgelegt wird, in der Regel mitgewährleistet ist. ${ }^{52}$ Das BVerfG kündigt jedoch auch an, die Grundrechte des Grundgesetzes - entsprechend der Vorgehensweise bezüglich der EMRK - im Lichte der GRCh auszulegen. ${ }^{53}$ Hinsichtlich des Prüfungsmaßstabs im Falle eines fehlenden Umsetzungsspielraums können die Grundrechte der GRCh somit dann hinzutreten und vom BVerfG zu überprüfen sein, wenn konkrete und hinreichende Anhaltspunkte vorliegen, dass durch Anwendung des GG das grundrechtliche Schutzniveau des Unionsrechts ausnahmsweise nicht gewährleistet ist. ${ }^{54}$ Dies wäre aus Sicht des BVerfG dann der Fall, wenn konkrete und hinreichende Anhaltspunkte dafür bestehen, dass das unionsrechtliche Fach-

46 EuGH, Rs. C-617/10 v. 26.2.2013, Rn. 29 - Fransson.

47 BVerfG, Beschluss des Ersten Senats vom 06. November 2019 - 1 BvR 16/13, Rn. 42.

48 Vgl. BVerfG a.a.O., Rn. 50 unter Berufung auf Präambel, Abs. 3 GRCh; Präambel, Abs. 6 EUV.

49 BVerfG, a.a.O., Rn. 42.

50 BVerfG, a.a.O., Rn. 43.

51 BVerfG, a.a.O., Rn. 43.

52 BVerfG, a.a.O., Rn. 55.

53 BVerfG, a.a.O., Rn. 60.

54 BVerfG, a.a.O., Rn. 63. 
recht für seine Durchführung trotz seiner Gestaltungsoffenheit ausnahmsweise engere grundrechtliche Maßgaben enthält, dass trotz zulässiger Grundrechtsvielfalt die Vermutung, nach der das Schutzniveau der GRCh durch die Anwendung der Grundrechte des Grundgesetzes mitgewährleistet ist, widerlegt sein könnte oder im konkreten Fall der Unionsgesetzgeber keine Grundrechtsvielfalt erreichen möchte. ${ }^{55}$

\section{Bedeutung für den Grundrechtsschutz}

Das BVerfG gibt mit seinen beiden Beschlüssen die strikte Trennung zwischen Unionsgrundrechten und Grundrechten des Grundgesetzes auf. Auch wenn das BVerfG augenscheinlich dennoch davon ausgeht, dass eine parallele Anwendung nationaler und europäischer Grundrechte grundsätzlich ausscheidet, so ist dieser Schluss nicht mehr ausnahmslos zwingend. Einerseits ist es in gewissen Konstellationen möglich, dass trotz eines mitgliedstaatlichen Umsetzungsspielraums die Unionsgrundrechte Anwendung finden können. Andererseits werden die Unionsgrundrechte im Falle der ausschließlichen Anwendung nationaler Grundrechte neuerdings als Auslegungshilfe einbezogen. Die vormals stringente Trennung der Grundrechtsebenen wurde somit aufgegeben. Dessen ungeachtet, war das Trennungsprinzip selbst bis dato Gegenstand erheblicher Kritik. Die strikte Trennung der Grundrechtsebenen ist praktisch mit erheblichen Problemen behaftet, da die Abgrenzung der Ebenen umso problematischer ist, wenn ein Sachverhalt teilweise unionsrechtlich determiniert ist, teilweise den Mitgliedstaaten einen Spielraum belässt. ${ }^{56}$

Das österreichische Verfassungsgericht begründete seine Zuständigkeit mit dem unionsrechtlichen Äquivalenz- und Effektivitätsgebot. ${ }^{57}$ Soweit das Unionsrecht, einschließlich der allgemeinen unionsrechtlichen Grundsätze, keine gemeinsamen Vorschriften zur Durchführung des Unionsrechts enthält, richten sich die Maßnahmen zur Durchführung von Unionsrechtsakten nach nationalem Recht. ${ }^{58}$ Dabei muss dieses so ausgestaltet und angewendet werden, dass die Wirksamkeit des Unionsrechts gewährleistet ist, und dass unionsrechtliche Sachverhalte nicht schlechter behandelt werden als rein nationale Konstellationen - sog. Effektivitäts- und Äquivalenzprinzip. Für gerichtliche Verfahren, die den Schutz der dem Bürger aus dem Unionsrecht erwachsenden Rechte gewährleisten sollen, bedeutet dies, dass sie nicht ungünstiger gestaltet sein dürfen als bei entsprechenden, rein nationalen Verfahren (Äquivalenzgrundsatz), und sie dürfen die Ausübung der Rechte nicht praktisch unmöglich machen oder übermäßig erschweren (Effektivitätsgrundsatz). ${ }^{59}$ Wollte das BVerfG auf die Argumentation des österreichischen Verfassungsgerichtshofs rekurrieren, so müsste im Lichte des

55 BVerfG a.a.O., Rn. $67 \mathrm{f}$.

56 Meyer/Hölscheidt/Schwerdtfeger, Charta der Grundrechte der Europäischen Union, 5. Aufl. (2019), Art. 51 GRCh Rn. 43.

57 Österreichischer Verfassungsgerichtshof v. 14.3.2012, U466/11 u.a.; kritisch dazu, Pöschl, ZöR 2012, 587.

58 EuGH v. 21.9.1983, Rs. 205/82, Slg. 1983, 2663, ECLI:EU:C:1983:233, Rn. 17 - Milchkontor; EuGH v. 1.12.1998, Rs. C-326/96, ECLI:EU:C:1998:577 - Levez, Slg. 1998, I-7835.

59 EuGH 1.12.1998, Rs. C-326/96, ECLI:EU:C:1998:577 - Levez, Slg. 1998, I-7835. 
Äquivalenzgrundsatzes eine Grundrechtskontrolle bei Fällen mit unionsrechtlichem Bezug genauso realisierbar sein, wie dies bei rein nationalen Sachverhalten möglich ist.

Hätte das BVerfG weiterhin auf eine Grundrechtskontrolle am Maßstab der GRCh verzichtet, hätten die Fachgerichte in Sachen Grundrechtsanwendung innerhalb des deutschen Justizsystems ,das letzte Wort“ gehabt, indem sie, auch soweit die Auslegung der Grundrechte geklärt ist, die Aufgabe haben, diese im Einzelfall anzuwenden und dementsprechend eine Abwägung kollidierender Grundrechtsbelange zu vollziehen. Das BVerfG selbst kann seine Rolle als Garant eines umfassenden innerstaatlichen Grundrechtsschutzes aber nur dann vollständig wahrnehmen, wenn es die Fachgerichte diesbezüglich vollumfänglich kontrollieren kann. Dabei untermauert das BVerfG seine eigene Prüfungskompetenz am Maßstab der Unionsgrundrechte mit der Funktionsähnlichkeit von Grundrechten der GRCh und denen des Grundgesetzes. Bliebe nur den Fachgerichten die Möglichkeit, ein deutsches Gesetzes bei einer Unionswidrigkeit unangewendet $\mathrm{zu}$ lassen, ${ }^{60}$ so würde das Verwerfungsmonopol des BVerfG entwertet. ${ }^{61}$ Das Verwerfungsmonopol aus Art. 100 GG dient der Schaffung von Rechtssicherheit und Vermeidung von Rechtszersplitterung ${ }^{62}$ und ist Bestandteil der grundgesetzlich ausgestalteten Gewaltenteilung. ${ }^{63}$ Gleiches sollte auch dann gelten, wenn die deutsche Staatsgewalt unionsrechtlich determiniert ist. Gleichwohl bleibt es problematisch, wenn die Prinzipien der grundgesetzlichen Gewaltenteilung angeführt werden, obwohl die Grundrechte des Grundgesetzes gar nicht Anwendung finden, sondern allein das Unionsrecht Prüfungsmaßstab bleibt. Dennoch erscheint es aufgrund des unionsrechtlichen Äquvialenz- und Effektivitätsgebot geboten, diese Maßstäbe entsprechend auf die Anwendung von determiniertem Unionsrecht seitens der deutschen Staatsgewalt zu übertragen. Auch mangels Individualrechtsschutz hinsichtlich der GRCh ist die Rechtsprechungsänderung des BVerfG begrüßenswert.

Gleichwohl geht das BVerfG mit seinen Entscheidungen nicht so weit, dass sich die EU-Grundrechte, kraft nationalen Rechts, außerhalb ihres in Art. 51 Abs. 1 GRCh festgelegten Anwendungsbereichs auf die Tätigkeiten nationaler Stellen auswirken. ${ }^{64}$

Sicherlich übte das BVerfG auch durch seine bisherige Trennungstheorie eine nicht unbedeutende Kontrollfunktion hinsichtlich der Unionsgrundrechte (bei vollständig determinierten Unionsakten) aus. Die Nichtvorlage eines Fachgerichts trotz entsprechender Pflicht (Art. 267 Abs. 3 AEUV) führte nach ständiger Rechtsprechung des BVerfG seit dem Solange II-Beschluss zu einem Entzug des gesetzlichen Richters nach Art. 101 Abs. 1 S. 2 GG und somit zu einem Verfassungsverstoß.65 Durch die Kontrolle der Fachgerichte bestand somit eine mittelbare Einflussmöglichkeit des BVerfG auf die Anwendung der Unionsgrundrechte, obgleich diese Konstruktion auf

60 Und nach Sicht des EuGH sogar müssen, Darüber hinaus ordnete der EuGH - wie bereits in der Mangold-Entscheidung - an, die Gerichte dürften die diskriminierende Regel auch tatsächlich nicht mehr anwenden, Wackerbarth/Kreße, EuZW 2010, 252 (252).

61 BeckOK Grundgesetz/Morgenthaler, 41. Ed. 15.2.2019, GG Art. 100 Rn. 2.

62 BeckOK Grundgesetz/Morgenthaler, 41. Ed. 15.2.2019, GG Art. 100 Rn. 2.

63 Maunz/Dürig/Dederer, Grungesetz Kommentar, 88. EL August 2019, Art. 100 GG, Rn. 15.

64 Jarass würde dies begrüßen. Jarass/Jarass GrCh, EU-Grundrechte-Charta, 3. Aufl. (2016), Art. 51 GRCh, Rn. 30aa.

65 BVerfGE 73, 339 (366); 126, 286 (315); 135, 155 (231). 
der Vorstellung beruhte, dass die Unionsgrundrechte selbst vor dem BVerfG nicht überprüfbar seien. Das BVerfG wiederum beschränkte sich unmittelbar auf eine Willkürkontrolle. ${ }^{66}$ Auch wenn dies, trotz der geänderten Rechtsprechung, auch heute noch einen Beschwerdegrund darstellt, ist durch die eigene Prüfungsbefugnis des BVerfG eine intensivere Kontrolle sichergestellt. Dies verdeutlicht schon die Einbeziehung in das Verfahren der Verfassungsbeschwerde und die Verbindlichkeit der Entscheidungen des BVerfG, $\S 31$ Abs. 1 BVerfGG. ${ }^{67}$

Die vorgenannten Beschlüsse führen insgesamt $\mathrm{zu}$ einem Einflussgewinn des BVerfG hinsichtlich der Auslegung und Anwendung von Unionsgrundrechten.

$\mathrm{Zu}$ beachten bleibt jedoch, dass der EuGH das Gericht mit der Letztentscheidungsbefugnis hinsichtlich der Auslegung und Anwendung der GRCh ist. Eine Gefahr der Missachtung dieser Stellung seitens des BVerfG ist nicht zu befürchten. Die Anwendung der GRCh durch das BVerfG wird stets durch den Dialog mit dem EuGH über das Institut des Vorabentscheidungsverfahrens begleitet werden (müssen). ${ }^{68}$ Das grundrechtlich erfahrene BVerfG kann hierzu einen wertvollen Beitrag leisten. ${ }^{69}$ Dies zeigen auch die Beispiele der Verfahren über den Ankauf von Staatsanleihen über $\mathrm{OMT}^{70}$ bzw. PSPP. In beiden rechtlichen Problemfeldern stärkte der Dialog mit dem EuGH das Verhältnis innerhalb des Europäischen Verfassungsgerichtsverbundes und stellte eine hohe Entscheidungsqualität sicher. Das BVerfG leistet auch mit den Rechtauf-Vergessen-Entscheidungen einen wichtigen Beitrag dazu, sich von dem Gedanken des Über- und Unterordnungsverhältnisses und den strikten Kompetenzverschränkungen zu lösen. Innerhalb des Europäischen Verfassungsgerichtsverbundes ist es notwendig, dass alle Beteiligten effektiv mitwirken. ${ }^{71}$ Der EuGH ist nämlich - auch aufgrund fehlenden Individualrechtsschutzes - auf geeignete Vorlagen angewiesen, um die Unionsgrundrechte näher zu konkretisieren. ${ }^{72}$ Die frühere Befürchtung seitens der deutschen Rechtswissenschaft, dass der EuGH Grundrechte nicht effektiv schütze, ist - auch aufgrund der primärrechtlichen Geltung der GRCh - heute nicht mehr haltbar. ${ }^{73}$ Das zeigt auch der Umstand, dass in der Solange-I-Entscheidung noch ein Grundrechtskatalog gefordert wurde, welcher schon in der Solange-II-Entscheidung nicht mehr explizit als notwendig angesehen wurde, heute aber dennoch existiert. ${ }^{74}$ Das BVerfG hat nun durch die Wahrnehmung des unionsgrundrechtlichen Schutzes die Möglichkeit, den Dialog der Grundrechtssysteme aktiv zu gestalten und Einfluss auf das europäische Wertesystem im Sinne der deutschen Grundrechte zu nehmen. ${ }^{75}$

66 Bäcker, EuR 2015, 389 (391), BVerfGE 129, 186, 204; dazu auch Sauer, EuZW 2011, 94 (96).

67 Bäcker, EuR 2015, 389 (401).

68 Bäcker, EuR 2015, 389 (405).

69 Bäcker, EuR 2015, 389 (405).

70 Es ist zu beachten, dass über OMT nie Staatsanleihenkäufe erfolgten, sondern nur die Ankündigung existierte, diese durchzuführen.

71 Etwa Voßkuhle, EuGRZ 2014, S. 165 (167).

72 Bäcker, EuR 2015, 389 (405).

73 Bäcker, EuR 2015, 389 (406).

74 BVerfGE 73, 339.

75 Griebel, Der Staat, Vol. 52 (2013), 371 (399). 
Im Rahmen des Kooperationsverhältnisses ${ }^{76}$ muss also auch das BVerfG seinen Beitrag dazu leisten, den europäischen Grundrechtschutz zu verstärken. ${ }^{77}$ Dieser Grundrechtsschutz spielt sich heute nicht mehr nur rein national ab, sondern aufgrund der Globalisierung und Entstehung von supranationalen Organisationen auch innerhalb des Mehrebenensystems. Mit dem durch das BVerfG mit seinem Solange-Rechtsprechung begründeten, eigentlich europarechtsfreundlichen judicial self-restraint hat sich das BVerfG zugleich in europarechtsunfreundlicher Weise der Möglichkeit beraubt, die unionsgrundrechtsgemäße Anwendung des EU-Rechts durch deutsche Behörden und Fachgerichte zu überprüfen. ${ }^{78}$ Damit beraubte es sich zugleich auch der Möglichkeit, einerseits die Integrationswirkung der GRCh zu verstärken und andererseits einen wirklich wirkmächtigen Platz im Europäischen Verfassungsgerichtsverbund einzunehmen. Das BVerfG erhält durch seinen Rechtsprechungswandel nun die Chance, rechtliche Unzulänglichkeiten im Dialog mit dem EuGH aufzudecken. ${ }^{79}$ Art. 23 Abs. 1 GG enthält die Verpflichtung aller Verfassungsorgane, auf die europäische Integration hinzuwirken, welche nicht im Belieben der politischen Akteure steht. ${ }^{80}$ Diese Aufgabe muss auch das BVerfG wahrnehmen - eine Aufgabe, die über die bloße Kontrolle der übrigen Verfassungsorgane hinausgeht.

Die bisherige Zurückhaltung in diesem Bereich verwundert, insbesondere im Vergleich zur EMRK. Hier hat das BVerfG die Fachgerichte schon über lange Zeit dahingehend kontrolliert, ob sie die EMRK richtig ausgelegt und angewandt haben. ${ }^{81}$

Insgesamt nehmen die Entscheidungen auch etwas Brisanz aus der Frage der Konturierung des Art. $51 \mathrm{GRCh}$, indem die EU-Grundrechte auch bei der Auslegung der nationalen Grundrechte miteinbezogen werden können.

Auch führt die Erstreckung des Prüfungsumfangs auf die Unionsgrundrechte dazu, dass die nationalen verfassungskulturellen Traditionen und Kontexte der jeweiligen Probleme ausreichend berücksichtigt werden können. Dies bestätigt einerseits die Rechtsprechung des EuGH, welche Verfassungs- und damit auch Grundrechtsvielfalt anerkennt. Weiterhin streitet dafür das Subsidiaritätsprinzip aus Art. 5 EUV. Auch die Vorabentscheidungsverfahren bilden dafür das richtige Mittel, welches ideal für den Dialog zwischen EuGH und BVerfG im Rahmen der Grundrechte ist. Der EuGH entscheidet mit dem Vorabentscheidungsverfahren im Grunde nicht über den Einzelfall, sondern ist Akteur in einem System der Kooperation und der Koordination zu den nationalen Gerichten. ${ }^{82}$ Art. 267 AEUV geht von der Prämisse aus, dass das mitgliedstaatliche Recht und das Unionsrecht zwei getrennte Rechtsordnungen bilden und die Kontrolle der Anwendung des Unionsrechts wegen der dezentralisierten Struktur der

76 So wird das Verhältnis aus Sicht des BVerfG und EuGH verstanden, vgl. BVerfG v. 15.12.2015 - 2 BvR 2735/14, Rn. 44; Voßkuhle, NVwZ 2010, 1 (1); Lenaerts, EuR 2015, 3 (25).

77 Griebel, Der Staat, Vol. 52 (2013), 371 (394).

78 Griebel, Der Staat, Vol. 52 (2013), 371 (394).

79 Ruffert, EuGRZ 2017, 241 (245).

80 BVerfGE 123, 267 (346 f).

81 Griebel, Der Staat, Vol. 52 (2013), 371 (381, 384).

82 Groeben, von der/Schwarze/Gaitanides, Europäisches Unionsrecht, 7. Aufl. (2015), Art. 267 AEUV, Rn. 4. 
Union in weiten Bereichen den nationalen Gerichten obliegt. ${ }^{83}$ Über das Vorabentscheidungsverfahren als Zwischenverfahren werden Auslegungs- und Gültigkeitsfragen des Unionsrechts vorab verbindlich geklärt, über Fragen hinsichtlich des innerstaatlichen Rechts darf der Gerichtshof jedoch nicht entscheiden. ${ }^{84}$ Sinn des Vorabentscheidungsverfahren ist es, die einheitliche Auslegung des Unionsrechts zu gewährleisten. ${ }^{85}$ Diesem Verfahren kommt damit eine zentrale Bedeutung für die Verwirklichung der Aufgabe des EuGH zu, die Wahrung des Rechts bei der Auslegung und Anwendung der Verträge sicherzustellen, Art. 19 Abs. 1 UAbs. 1 S. 2 EUV. ${ }^{86}$ Dazu ist es aber unumgänglich, dass alle wesentlichen Fragen, die die Auslegung des Unionsrechts betreffen, durch den EuGH geklärt werden.

Entsprechend seiner Aufgabe innerhalb des Vorabentscheidungsverfahrens wird der EuGH stets Ansätze wählen, die auf alle Mitgliedstaaten übertragbar sind. Damit ist sichergestellt, dass Entscheidungen auch auf die Rechtsordnungen der nicht am Verfahren beteiligten Mitgliedstaaten anwendbar sind. Die konkrete Anwendung obliegt aufgrund des Charakters als Zwischenverfahren den mitgliedstaatlichen Gerichten. An dieser Anwendung muss das BVerfG als nationales Verfassungsgericht mit seinen Entscheidungen in entscheidungserheblicher Wirkung Einfluss nehmen können.

\section{Verhältnis zu Solange II}

Kritisch zu beleuchten ist die bemerkenswerte Aussage des Ersten Senates hinsichtlich des Verhältnisses zur Solange-II-Entscheidung des Zweiten Senats aus dem Jahre 1986. Wörtlich heißt es:

„Das Bundesverfassungsgericht hat daher entscheidungserheblich jeweils lediglich zum Ausdruck gebracht, seine Gerichtsbarkeit über die Anwendbarkeit von abgeleitetem Gemeinschafts- beziehungsweise Unionsrecht, das innerstaatliche Behörden und Gerichte als Rechtsgrundlage für ihr Handeln herangezogen haben, nicht auszuüben und dieses Recht nicht an den Grundrechten des Grundgesetzes zu prüfen (vgl. nur BVerfGE 73, $339<387>$; 102, $147<163>$ ).

Demgegenüber geht es in der vorliegenden Entscheidung nicht um eine Infragestellung von Unionsrecht, sondern um dessen richtige Anwendung im Lichte des klaren oder geklärten Gehalts der Unionsgrundrechte. Hierzu enthält die bisherige Rechtsprechung des Zweiten Senats weder ausdrücklich noch implizit eine Aussage. " 87

Damit begründet der Erste Senat seine Entscheidung, nicht das Plenum (§ 16 I BVerfGG) anrufen zu müssen. Dies ist immer dann notwendig, wenn ein Senat von der in einer Entscheidung des anderen Senats enthaltenen Rechtsauffassung abweichen will. Zutreffend ist hierbei grundsätzlich, dass sich die Überprüfung einer

83 Grabitz/Hilf/Nettesheim/Karpenstein, Das Recht der Europäischen Union, 68. EL Oktober 2019, Art. 267 AEUV, Rn. 2.

84 Streinz/Ehricke, EUV/AEUV, 3. Aufl. (2018), Art. 267 AEUV, Rn. 7.

85 Groeben, von der/Schwarze/Gaitanides, Europäisches Unionsrecht, 7. Aufl. (2015), Art. 267 AEUV, Rn. 10.

86 Streinz/Ehricke, EUV/AEUV, 3. Aufl. (2018), Art. 267 AEUV, Rn. 6.

87 BVerfG, Beschluss des Ersten Senats vom 06. November 2019 - 1 BvR 276/17, Rn. 90. 
Rechtsgrundlage auf ihre Vereinbarkeit mit Grundrechten von dem Fall unterscheidet, bei dem es fraglich ist, ob Unionsgrundrechte bei der Anwendung von Unionsakten gewahrt wurden. Es gibt jedoch Stimmen in der Literatur, die die Solange-Rechtsprechung weit auslegen und auch Konstellationen erfasst sehen, in denen die Auslegung und Anwendung eines Unionsrechtsakts in Rede steht, der für sich genommen zweifellos grundrechtskonform ist. ${ }^{88}$ Die Auslegung und Anwendung des Unionsrechts seien an sich rein unionsrechtliche Vorgänge, weshalb grundrechtliche Direktiven hierfür allein aus den Unionsgrundrechten, nicht aber aus den Grundrechten des Grundgesetzes zu entwickeln seien. ${ }^{89}$ Selbst wenn dies zutreffend ist, ${ }^{90}$ so geht es hier nicht um die Anwendung von Grundrechten des Grundgesetzes oder die mittelbare Überprüfbarkeit von Unionsrecht, sondern um die Anwendung von Unionsrecht im Einzelfall und der Wahrung des Grundrechtsschutzes durch die GRCh. Gegenstand der Verfassungsbeschwerde ist also die Kontrolle einer Entscheidung eines deutschen Fachgerichts daraufhin, ob es bei der ihm obliegenden Anwendung des Unionsrechts den hierbei zu beachtenden Anforderungen der Charta Genüge getan hat. ${ }^{91}$

\section{Fazit}

Sicherlich hat sich das BVerfG durch die Inanspruchnahme seiner Kontrollfunktion von der vormals strikten Trennungstheorie gelöst. Die möglicherweise vorhandene prima-facie Annahme, der Erste Senat habe sich zugleich von der Solange-II-Rechtsprechung losgesagt, ist insbesondere vor dem Hintergrund, dass das BVerfG nicht auf seine sog. „Reservekompetenz" verzichtet hat, nicht haltbar. Demnach bleibt es bei dem Grundsatz, dass die Anwendung von Unionsrecht nicht an den Grundrechten des Grundgesetzes gemessen wird. Für den Ausnahmefall behält sich das BVerfG jedoch weiterhin vor, eine Prüfung am Maßstab der Grundrechte des Grundgesetzes anzustellen. Dies käme - entsprechend des Solange-II-Vorbehalts - allerdings nur dann zum Tragen, wenn der Grundrechtsschutz, wie er vom EuGH konkretisiert, durch die GRCh gewährleistet und nunmehr vom BVerfG herangezogen wird, in einer, nicht den Voraussetzungen des Art. 23 I GG entsprechenden Weise hinter dem nationalen Grundrechtsschutz generell zurückbleibt. In einem so gelagerten Fall würde der Solange-II-Vorbehalt folgerichtig wieder aufleben. Demnach ist in den Beschlüssen des BVerfG nicht die Aufgabe, sondern eine Fortentwicklung der Solange-II-Rechtsprechung zu erblicken, die mit Recht eine Wertigkeit einnimmt, die sich als ,Solange III“ bezeichnen lässt.

Schwieriger gestaltet sich jedoch weiterhin das Verhältnis zur (menschenwürderelevanten) Identitätskontrolle. Zwar geht das BVerfG strukturell nicht von einer Entfaltung der Grundrechtskontrolle über die „Hintertür“ der Identitätskontrolle aus, sondern trennt zwischen beiden Vorbehalten über das Kriterium der Verfassungsidentität.

88 Bäcker, EuR 2015, 389 (395); Griebel, Der Staat, Vol. 52 (2013), 371 (386).

89 Bäcker, EuR 2015, 389 (395); Griebel, Der Staat, Vol. 52 (2013), 371 (386).

90 Die Kritik ist im Wortlaut der Entscheidung in Solange II - Rn. 132 zu sehen, welche nur von der „Anwendbarkeit von abgeleitetem Gemeinschaftsrecht, das als Rechtsgrundlage [...] in Anspruch genommen wird." spricht.

91 BVerfG, a.a.O., Rn. 52. 
Dennoch bestehen Überschneidungen zwischen beiden Instituten. Gleichwohl bleibt das Verhältnis dieser Instrumente zueinander gegenwärtig ungeklärt. Hier bleibt abzuwarten, inwieweit künftige Entscheidungen des BVerfG - im Lichte des nun gewandelten Verständnisses des Ersten Senates - eine weitergehende Konkretisierung vornehmen werden. 